\title{
J.-P. BOREL
}

\section{F. LAUBIE}

\section{Quelques mots sur la droite projective réelle}

Journal de Théorie des Nombres de Bordeaux, tome 5, no 1 (1993), p. $23-51$

<http://www.numdam.org/item?id=JTNB_1993_5_1_23_0>

(C) Université Bordeaux 1, 1993, tous droits réservés.

L'accès aux archives de la revue «Journal de Théorie des Nombres de Bordeaux » (http://jtnb.cedram.org/) implique l'accord avec les conditions générales d'utilisation (http://www.numdam.org/conditions). Toute utilisation commerciale ou impression systématique est constitutive d'une infraction pénale. Toute copie ou impression de ce fichier doit contenir la présente mention de copyright.

\section{Numdam}

Article numérisé dans le cadre du programme

Numérisation de documents anciens mathématiques

http://www.numdam.org/ 
Journal de Théorie des Nombres

de Bordeaux 5 (1993), 23-51

\title{
Quelques mots sur la droite projective réelle
}

\author{
par J.-P. Borel et F. Laubie
}

\section{Introduction}

Étant donné un nombre réel $\theta$, la suite $([n+1) \theta]-[n \theta]-[\theta])$ souvent appelée mot caractéristique de $\theta$ est formée de 0 et de 1 . Elle a été étudiée par de très nombreux auteurs depuis Bernoulli en 1772 [1] et Christoffel en 1875 [7] jusqu'à S. Ito et S. Yasutomi en 1990 [9] et T. C. Brown en 1991 [4]. Ces derniers caractérisent les mots caractéristiques des nombres irrationnels comme des points fixes de certaines substitutions.

En fait ce résultat trouve sa vraie place dans une théorie fondée sur les suites de Farey, la représentation bien connue de Félix Klein des fractions continues ainsi que sur la factorisation des monoïdes libres par les mots de Lyndon (voir, par exemple, [12] ou [16]) ; cela consiste à identifier la droite projective réelle $\mathbb{P}^{1}$ à un ensemble $\mathbb{L}$ de suites de 0 et 1 appelées mots de Christoffel de sorte que l'action des homographies de $P G L_{2}(\mathbb{Z})$ sur $\mathbb{P}^{1}$ corresponde à celle de certaines substitutions sur $\mathbb{L}$.

L'objet de cet article est d'exposer cette théorie et quelques applications arithmétiques avec des preuves complètes. La plupart des résultats que nous présentons ont été annoncés dans deux notes aux Compte-Rendus de l'Académie des Sciences de Paris ([3] et [10]). Dans [10], le théorème à l'origine de ce travail, qui caractérise les nombres quadratiques comme les pentes des mots de Christoffel infinis fixes sous l'action de certaines substitutions (voir le corollaire au théorème 5 ci-dessous), a été attribué à T. C. Brown. En fait ce résultat avait été établi (sous une forme assez différente, il est vrai) par S. Ito et S. Yasutomi deux ans auparavant [9]. Il a été plusieurs fois réétudié depuis ([5], [6], [11]).

Dans une première partie, nous présentons les mots de Christoffel primitifs finis ou si l'on préfère les plus petites périodes des mots caractéristiques des nombres rationnels. Nous montrons que ce sont en fait les mots de Lyndon maximaux. À ce titre ils jouissent d'une factorisation en mots de

Manuscrit reçu le 28 octobre 1991.

Les résultats contenus dans cet article ont été exposés au colloque "Thémate", (CIRM, Luminy, Mai 1991). 
Lyndon dite factorisation standard [12]. Nous montrons que cette factorisation standard est leur unique factorisation en mots de Christoffel primitifs. Il en découle une construction systématique des mots de Christoffel qui est analogue à la partie "finitiste" de la construction de Rauzy [14] ou encore à la construction de Raney [13].

Dans la deuxième partie, on étudie les mots de Christoffel primitifs infinis. Leur construction, qui est un prolongement naturel du cas fini, est analogue à celle des mots sturmiens proposée par Rauzy [14] ; elle fournit le développement en fraction continue de leurs pentes. On peut identifier l'ensemble $\mathbb{L}^{+}$des mots de Christoffel infinis primitifs commençant par 0 , muni de l'ordre lexicographique induit par $0<1$, à l'ensemble ordonné $\mathbb{R}^{+}$des nombres réels positifs. Autrement dit un mot de Christoffel infini primitif peut être considéré comme une écriture particulière d'un nombre réel.

La troisième et dernière partie traite des substitutions de mots de Christoffel, et en particulier d'une classe de substitutions dites standard, qui jouent sur l'ensemble des mots de Christoffel le rôle des homographies $\frac{a x+b}{c x+d}(a, b, c, d \in \mathbb{Z}$ et $a d-b c= \pm 1)$ sur la droite projective réelle. Ainsi les propriétés classiques du groupe modulaire ont une traduction en termes de substitutions de mots de Christoffel. De plus, cette théorie met en évidence les rapports entre les nombres quadratiques et les mots de Christoffel fixes par certaines de ces substitutions, ce qui redonne le théorème de Ito-Yasutomi [9]. Enfin, en application de ceci, nous proposons une élégante démonstration combinatoire de l'existence de solutions non triviales des équations de Pell-Fermat, due essentiellement à J. Riss [15].

Les auteurs tiennent à remercier les professeurs J. Shallit de l'Université de Waterloo pour son aide et J. Riss de l'université de Bordeaux car nous nous sommes largement inspirés de son travail original sur les suites de Farey [15].

\section{Mots de Christoffel et mots de Lyndon}

\section{Mots de Christoffel}

Dans cet article, $X$ désigne l'alphabet $\{0,1\}$ et $X^{*}$ le monoïde libre engendré par $X$. Si $f$ est un mot non vide appartenant à $X^{*}$, on note $|f|_{0}$ (resp. $|f|_{1}$ ) le nombre d'occurrences de 0 (resp. de 1 ) dans $f,|f|=$ $|f|_{0}+|f|_{1}$ la longueur de $f$ et $r(f)=|f|_{1} /|f|_{0} \in \mathbb{Q} \cup\{+\infty\}$. Si $f=u v$ où $u$ et $v$ sont des mots de $X^{*}$ on dit que $u$ est un préfixe de $f$ et que $v$ en est un suffixe ; pour tout entier $n \geq 1$, on note $u^{n}=u \ldots u$ avec $n$ facteurs $u$; on désigne par $u^{\infty}$ le mot infini périodique de période $u$. On dit que le 
mot $f \in X^{*}$ est primitif si $f$ est distinct de tous les mots $u^{n}$ avec $u \in X^{*}$ et $n$ entier $\geq 2$. Enfin, pour tout $f \in X^{*}$, on désigne par $-f$ le mot $f$ lu à l'envers.

Définitions. Un mot $f \in X^{*}$ est dit de Christoffel si pour tout préfixe $f^{\prime}$ de $f$ on a :

$$
r\left(f^{\prime}\right)=\max \left\{r(u) ; u \in X^{*},|u|=\left|f^{\prime}\right| \text { et } r(u) \leq r(f)\right\} .
$$

Dans la suite, de tels mots de Christoffel $f$ seront qualifiés de positifs, alors que ces mêmes mots lus à l'envers, $-f$, seront appelés mots de Christoffel négatifs.

Exemple. Pour tous entiers positifs $p$ et $q$ premiers entre eux, le mot $a_{1} a_{2} \ldots a_{q} \in X^{*}$ avec $a_{n}=\left[n \frac{p}{q}\right]-\left[(n-1) \frac{p}{q}\right]-\left[\frac{p}{q}\right]$ est un exemple de mot de Christoffel [7] dont l'étude par Jean Bernoulli remonte à 1772 [1].

Notations et Conventions. Désormais pour tout mot de Christoffel $f$, on note $f_{1}=|f|_{1}$ si $f$ est positif, $f_{1}=-|f|_{1}$ si $f$ est négatif, dans tous les cas $f_{0}=|f|_{0}$ et $\rho(f)=f_{1} / f_{0}$; on dit que $\rho(f)$ est la pente de $f$. Les seuls mots de Christoffel à la fois positifs et négatifs sont 0 et 1 ; dans la suite le symbole $1_{1}$ prendra la valeur +1 si 1 est considéré comme un mot de Christoffel positif et -1 dans l'autre cas ; il ne sera utilisé que lorsqu'aucune ambiguité ne sera posssible.

\section{Illustration géométrique}

Elle consiste à associer à tout mot $f$ dans $X^{*}$ un "chemin dans la grille" $\mathbb{N} \times \mathbb{R}^{+} \cup \mathbb{R}^{+} \times \mathbb{N}$ dessinée dans le plan : à chaque occurrence de 0 dans $f$ correspond un pas horizontal dirigé vers la droite et à chaque occurrence de 1 , un pas vertical dirigé vers le haut.

Soit $r \in \mathbb{R}^{+} \cup\{+\infty\}$; on désigne par $F_{r}$ l'ensemble des mots $f$ non vides tels que $r\left(f^{\prime}\right) \leq r$ pour tout préfixe non vide $f^{\prime}$ de $f$; ce sont exactement les mots dont le chemin est situé au-dessous de la droite vectorielle de pente $r$ dans $\mathbb{R}^{2}$. Si $r \in \mathbb{Q}^{+} \cup\{+\infty\}$, parmi les chemins des mots de $F_{r}$, les chemins les plus proches des segments de droites joignant leurs extrémités, (c'està-dire ceux pour lesquels les domaines ouverts compris entre eux-même et les segments joignant leurs extrémités ne contiennent pas de points entiers, i. e. à coordonnées entières), sont les chemins des mots de Christoffel de pente $r$. 
En ce qui concerne les mots de Christoffel négatifs, on les représente par des chemins dans la "grille" $\mathbb{N} \times \mathbb{R}^{-} \cup \mathbb{R}^{+} \times \mathbb{Z}^{-}$: à chaque occurrence de 0 correspond toujours un pas horizontal dirigé vers la droite mais à chaque occurrence de 1 correspond un pas vertical dirigé vers le bas. Ainsi, si $f$ est un mot de Christoffel négatif sa pente est $\rho(f)=-r(f)=-r(-f)=$ $-\rho(-f)$.

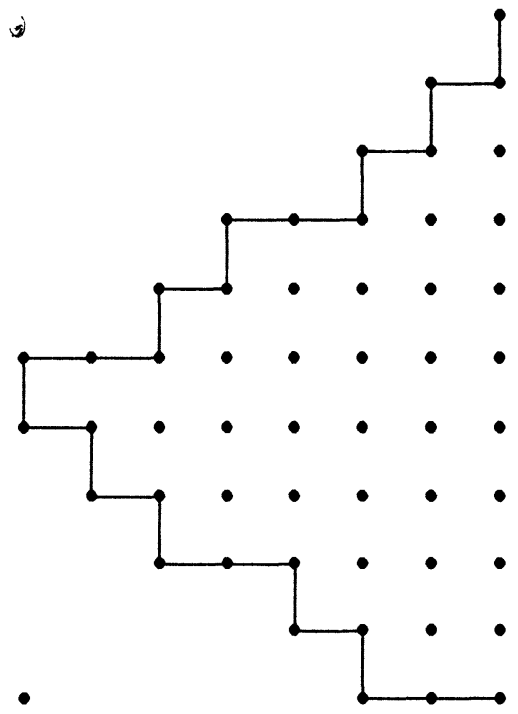

001010010101 est un mot de Christoffel positif de pente $\frac{5}{7}$ alors que 101010010100 est un mot de Christoffel négatif de pente $\frac{-5}{7}$.

Figure 1

Dans la suite, nous utiliserons souvent le :

Lemme. Pour tous mots non vides $u$ et $v$, on a :

$$
\min (r(u), r(v)) \leq r(u v) \leq \max (r(u), r(v))
$$

avec égalités si et seulement si $r(u)=r(v)$.

L'interprétation géométrique rend ce résultat suffisamment évident. 


\section{Mots de Christoffel primitifs}

Munissons l'ensemble $X^{*} \backslash\{\emptyset\}$ de l'ordre lexicographique induit par $0<$ 1. Étant donnés deux mots $f$ et $g$ tels que $f<g$ et que $f$ ne soit pas un préfixe de $g$, il existe un mot $m \in X^{*}$ tel que $m 0$ soit un préfixe de $f$ et $m 1$ un préfixe de $g$; si $f$ est un mot de Christoffel positif de pente $\rho$, alors $\rho(m 0) \leq \rho<\rho(m 1)$, donc $g \notin F_{\rho}$ et comme $f<m 1 \leq g$, on en déduit que $f$ est le plus grand des mots de $F_{\rho}$ de longueur $|f|$. Les propriétés suivantes en résultent aussitôt :

1. Étant donnés deux mots de Christoffel de même pente, l'un d'eux est un préfixe de l'autre.

2. Étant donné un couple d'entiers naturels $(p, q)$, il existe un unique mot de Christoffel $f$ de pente $\frac{p}{q}$ et de longueur $p+q$. En outre, si $p=0$ on pose $d=q, p^{\prime}=0$ et $q^{\prime}=1$; si $q=0$ on pose $d=p, q^{\prime}=0$ et $p^{\prime}=1$; si $p$ et $q$ sont non nuls, on note $d$ leur p.g.c.d. et on pose $p=d p^{\prime}$ et $q=d q^{\prime}$; dans ces conditions, le mot de Christoffel $g$ de pente $\frac{p^{\prime}}{q^{\prime}}$ et de longueur $p^{\prime}+q^{\prime}$ est primitif et $f=g^{d}$.

3. Les mots de Christoffel primitifs sont les mots de Christoffel $f$ pour lesquels $\rho\left(f^{\prime}\right)<\rho(f)$ quel que soit le préfixe strict non vide $f^{\prime}$ de $f$ ou encore pour lesquels $\rho\left(f^{\prime \prime}\right)>\rho(f)$ quel que soit le suffixe strict non vide $f^{\prime \prime}$ de $f$.

4. Si $f$ est un mot de Christoffel positif avec $r=\rho(f)$ alors $f$ est un préfixe de tous les mots de $F_{r}$ qui lui sont supérieurs.

5. Étant donnés deux mots $f$ et $g$ tels que $f<g$, si $f$ est un mot de Christoffel positif, alors il existe un préfixe $g^{\prime}$ de $g$ tel que $f=g^{\prime}$ ou tel que $f<g^{\prime}$ et $\rho(f)<\rho\left(g^{\prime}\right)$.

6. Si $f$ et $g$ sont deux mots de Christoffel primitifs positifs alors : $f<g \Leftrightarrow \rho(f)<\rho(g)$.

\section{Mots de Lyndon maximaux}

On rappelle qu'un mot de Lyndon de $X^{*}$ relatif à un ordre lexicographique sur $X^{*}$ est un mot qui, pour cet ordre, est inférieur à tous ses suffixes. De plus un mot de Lyndon $f$ distinct de 0 et de 1 peut s'écrire $f=u v$ où $u$ et $v$ sont eux-même des mots de Lyndon ; quand $v$ est choisi de longueur maximale, on note $v=f_{\delta}, u=f_{\gamma}$ et l'expression $f=f_{\gamma} f_{\delta}$ est connue sous le nom de factorisation standard de $f$ [12]. 
ThÉORÈme 1. Soit $r \in \mathbb{Q}^{+} \cup\{+\infty\}$ (resp. soit $r \in \mathbb{Q}^{-} \cup\{-\infty\}$ ). Le mot de Christoffel primitif de pente $r$ est le mot de Lyndon maximal parmi les mots de Lyndon de pente $r$ pour l'ordre lexicographique sur $X^{*}$ induit par $0<1$ (resp. induit par $1<0$ ) ; de plus sa factorisation standard en est l'unique factorisation en mots de Christoffel primitifs.

Preuve. Il suffit clairement de limiter la preuve aux mots de Christoffel positifs. Soit donc $f$ l'unique mot de Christoffel primitif de pente $r \geq 0$ (voir la propriété 2 ci-dessus). Soient $f^{\prime}$ et $f^{\prime \prime}$ des mots non vides tels que $f=f^{\prime} f^{\prime \prime}$; tout préfixe de $f$ et donc aussi de $f^{\prime} f$ est de pente $<r$; si $f<f^{\prime} f$ alors d'après la propriété $4, f$ serait un préfixe de $f^{\prime} f$ donc $f^{\prime \prime}$ serait un préfixe de $f$ ce qui est impossible puisque $\rho\left(f^{\prime \prime}\right)>\rho(f)$; on a $f>f^{\prime} f$ ou encore $f^{\prime \prime}>f$, ce qui prouve que $f$ est un mot de Lyndon.

Soit $g$ un autre mot de Lyndon de pente $r$ et montrons que $f>g$. Supposons que $f \leq g$; d'après la propriété 5 précédente, il existe un préfixe $g_{1}$ de $g$ tel que $g_{1}=f$ ou alors $g_{1}>f$ et $\rho\left(g_{1}\right)>r$; dans tous les cas, le mot $h_{1}$ tel que $g=g_{1} h_{1}$ vérifie $h_{1}=\emptyset$ ou $g<h_{1}$ et donc $f<h_{1}$; si $h_{1}=\emptyset$, soit $g_{2}$ un préfixe de $h_{1}$ tel que $\rho\left(g_{2}\right) \geq r$, (avec égalité seulement si $g_{2}=f$ ), et soit $h_{2} \in X^{*}$ tel que $h_{1}=g_{2} h_{2} ;$ on a alors $g=g_{1} g_{2} h_{2}$ avec $h_{2}=\emptyset$ ou $g<h_{2} \ldots$ et ainsi de suite, si bien que $g$ s'écrit :

$$
g=g_{1} g_{2} \cdots g_{k} \text { avec } \rho\left(g_{1}\right), \rho\left(g_{2}\right), \cdots, \rho\left(g_{k}\right) \geq r .
$$

Pour que $g$ soit de pente $r$ il faudrait que $\rho\left(g_{1}\right)=\rho\left(g_{2}\right)=\cdots=\rho\left(g_{k}\right)=r$ c'est-à-dire que $g_{1}=g_{2}=\cdots=g_{k}=f$, donc $g=f^{k}$, qui n'est pas un mot de Lyndon sauf si $k=1$. Supposons maintenant que le mot de Christoffel primitif positif $f$ est distinct de 0 et de 1 . Soit $f_{\gamma}$ le préfixe strict de longueur minimale tel que $\rho\left(f_{\gamma}\right)=\max \rho(g), g$ parcourant l'ensemble des préfixes stricts non vides de $f$; c'est clairement un mot de Christoffel primitif. On note $f_{\delta}$ le suffixe de $f$ tel que $f=f_{\gamma} f_{\delta}$; on sait que $\rho\left(f_{\delta}\right)>\rho(f)$. Soit $u$ un préfixe strict non vide de $f_{\delta}$; comme $\rho\left(f_{\gamma} u\right) \leq \rho\left(f_{\gamma}\right)$ on a $\rho(u) \leq \rho\left(f_{\gamma}\right)$ mais $\rho\left(f_{\gamma}\right)<\rho\left(f_{\delta}\right)$; donc $\rho(u)<\rho\left(f_{\delta}\right)$; si maintenant $v$ est un mot de même longueur que $u$ tel que $\rho(v)>\rho(u)$ alors $\rho\left(f_{\gamma} u\right)<\rho\left(f_{\gamma} v\right)$ et comme $f$ est de Christoffel $\rho\left(f_{\gamma} v\right)>\rho(f)=\rho\left(f_{\gamma} f_{\delta}\right)$ donc, puisque $|v|<\left|f_{\delta}\right|, \rho(v)>\rho\left(f_{\delta}\right)$; $f_{\delta}$ est donc un mot de Christoffel primitif.

L'interprétation géométrique des mots de Christoffel primitifs $f$ est particulièrement pertinente pour trouver l'unicité de la factorisation $f=f_{\gamma} f_{\delta}$ où $f_{\gamma}$ et $f_{\delta}$ sont également des mots de Christoffel primitifs. 


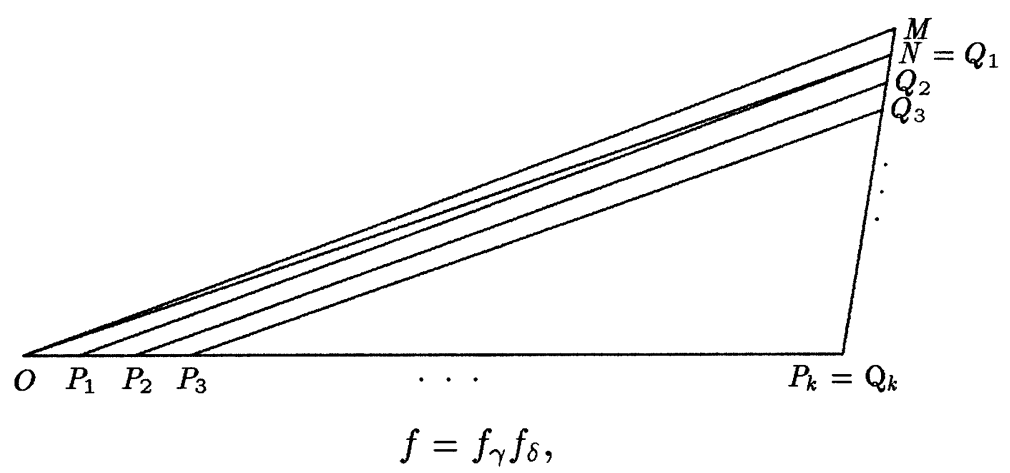

$f, f_{\gamma}$ et $f_{\delta}$ ont respectivement $O, O, N$ pour origines, et $M, N, M$ pour extrémités. $P_{1} N, P_{2} Q_{2}, P_{3} Q_{3} \cdots$ sont parallèles à $O M$ et équidistantes. (Pour la clarté de la figure, le repère n'est pas normé, en fait : $O P_{k} \leq 1$ et $M N \geq 1)$.

Figure 2

On sait déjà que $f$ n'admet pas de suffixes non vides $h$ tels que $|h|<\left|f_{\delta}\right|$ et $\rho(h)<\rho\left(f_{\gamma}\right)$. Il s'agit donc de prouver qu'il n'y a pas non plus de suffixes non vides $h$ de $f$ tels que $|h|>\left|f_{\delta}\right|$ et $\rho(h)<\rho\left(f_{\delta}\right)$ ou, ce qui revient au même, que sur la figure ci-dessus, le domaine triangulaire fermé $O M P_{k}$ ne contient pas d'autres points entiers que $O, M, N, Q_{2}, Q_{3}, \cdots, Q_{k}=$ $P_{k}$. Or on sait déjà que les seuls points entiers du domaine fermé $O M N$ sont $O, M$ et $N$; et donc qu'il y a pas d'autre point entier $S$ dans le domaine fermé $O N P_{1}$, car alors $N+\overrightarrow{S O}$ serait un point entier dans $O M N$. Maintenant s'il y avait un point entier $T$ autre que $Q_{i}$ ou $Q_{i+1}$ dans le domaine fermé $Q_{i} P_{i} Q_{i+1} P_{i+1}$ alors $Q_{i-1}+\overrightarrow{Q_{i} T}$ serait un point entier dans $Q_{i-1} P_{i-1} Q_{i} P_{i}$; ce qui prouve notre assertion par récurrence.

\section{Intervalles standard}

Étant donné un nombre rationnel $x$, convenons d'appeler $x=\frac{p}{q}$ l'écrituré canonique de $x$ si $p \in \mathbb{Z}, q \in \mathbb{N} \backslash\{0\}$ et si $p$ et $q$ sont premiers entre eux ; l'écriture canonique de $+\infty$ sera $\frac{1}{0}$ et celle de $-\infty, \frac{-1}{0}$. 
Définitions. Étant donnés deux mots de Christoffel primitifs positifs, (resp. négatifs), $f$ et $g$, l'ensemble des mots de Christoffel primitifs $h$ tels que $f \leq h \leq g$ pour l'ordre lexicographique sur $X^{*}$ induit par $0<1$ (resp. induit par $1<0)$ s'appelle un intervalle et est noté $(f, g)$; l'intervalle $(f, g)$ est dit standard si, les pentes de $f$ et $g$ étant écrites sous forme canonique $\rho(f)=\frac{p}{q}, \rho(g)=\frac{r}{s}$, on a $q r-p s=1$, c'est-à-dire si $[\rho(f), \rho(g)]$ est un intervalle de Farey (voir par exemple [8], ch. III).

Interprétation géométrique. Étant donnés deux mots de Christoffel primitifs $f$ et $g$ on appelle déterminant du couple $(f, g)$ et on note det $(f, g)$, l'aire algébrique du domaine limité par les chemins des mots $f g$ et $g f$ en comptant positivement l'aire des carrés situés au-dessus du chemin de $f g$ et négativement l'aire des autres. En fait, $|\operatorname{det}(f, g)|$ est égal à l'aire du parallélogramme $P(f, g)$ dont les sommets sont l'origine et les extrémités des chemins de $f, g$ et $f g$. C'est aussi le nombre de points entiers du tore dont $P(f, g)$ est le domaine fondamental. La démonstration de tout ceci est bien classique : il suffit de remarquer que det $(.,$.$) est une forme \mathbb{Z}$-linéaire alternée en ce sens que, pour tous mots de Christoffel primitifs $u, v$ et $w$ on a :

$$
\begin{aligned}
\operatorname{det}(u, v) & =-\operatorname{det}(u, v), \\
\operatorname{det}(u v, w) & =\operatorname{det}(u, w)+\operatorname{det}(v, w) .
\end{aligned}
$$

Autrement dit, en notant les pentes de $f$ et de $g$ sous forme canonique : $\rho(f)=\frac{p}{q}$ et $\rho(g)=\frac{r}{s}$, on a det $(f, g)=q r-p s$. En particulier, si l'intervalle $(f, g)$ est standard, alors les points entiers situés sur les chemins de $f g$ d'une part et de $g f$ d'autre part coïncident à l'exception d'un seul d'entre eux.

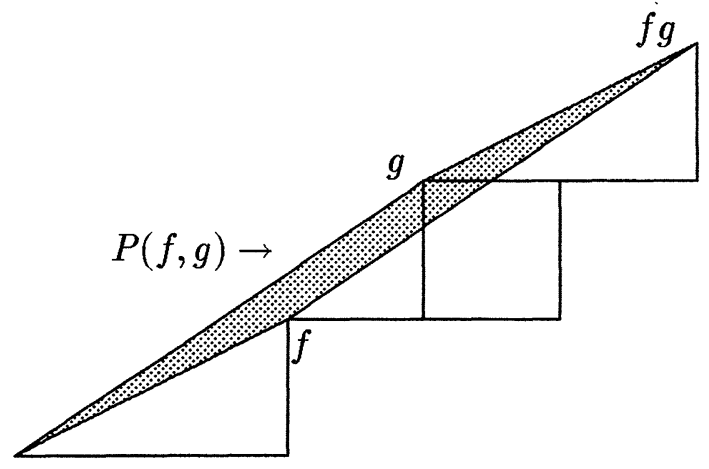

si $f=001$ et $g=00101$, alors $\operatorname{det}(f, g)=+1$.

Figure 3 


\section{Propriétés et définitions.}

Proposition 1 et définition. Étant donnés deux mots de Christoffel primitifs $f$ et $g$, l'intervalle $(f, g)$ est standard si et seulement si $f g$ est un mot de Christoffel primitif ; $f g$ s'appelle alors le mot médian de l'intervalle standard $(f, g)$.

Preuve. Compte tenu de ce que $-f g=(-g)(-f)$, il suffit de prouver la proposition 1 pour les mots de Christoffel positifs. Soient $f$ et $g$ deux mots de Christoffel primitifs positifs. Remarquons d'abord que si $f g$ est un mot de Christoffel primitif alors il est déjà écrit sous forme de sa factorisation standard, en particulier on a alors $f<g$. Considérons les chemins des mots $f$ et $g$, les segments de droite joignant les extrémités de ces chemins et le parallélogramme $P(f, g)$ construit sur ces deux segments. Dire que l'intervalle $(f, g)$ est standard c'est dire que le chemin $f g$ passe par tous les points entiers du chemin de $g f$ à l'exception d'un seul de ceux-ci qui se trouve être l'extrémité du chemin de $g$ c'est-à-dire un sommet du parallélogramme $P(f, g)$, (voir figure 3). Comme $f$ et $g$ sont des mots de Christoffel primitifs, il en résulte que det $(f, g)=+1$ si et seulement si l'intérieur du parallélogramme $P(f, g)$ ne contient pas de points entiers c'est-à-dire si et seulement si le mot $f g$ est un mot de Christoffel primitif.

Corollaire 1. Si $m$ est un mot de Christoffel primitif de l'intervalle standard $(f, g)$ distinct de $f$ et $g$ alors $|m| \geq|f|+|g|$.

Preuve. En effet le parallélogramme $P(f, g)$ de la preuve de la proposition 1 , ne contient pas de point entier dans son intérieur.

Corollaire 2. Si $(f, g)$ est un intervalle standard alors $(f g, g)$ et $(f, f g)$ sont aussi des intervalles standard.

Preuve. En effet si les points entiers des chemins des mots $f g$ et $g f$ coïncident à l'exception d'un seul, il en est de même pour les chemins des mots $f f g$ et $f g f$ d'une part et des mots $f g g$ et $g f g$ d'autre part.

Notations. Si $I=(f, g)$ est un intervalle standard, on note $I^{-}=(f, f g)$ et $I^{+}=(f g, g)$; soit $\omega=\omega_{1} \omega_{2} \cdots \omega_{n}$ avec $\omega_{i} \in\{+,-\}$ une suite finie de signes, on définit par récurrence l'intervalle standard $I^{\omega}$ en posant :

$$
I^{\omega}=\left(I^{\omega_{1} \cdots \omega_{n-1}}\right)^{\omega_{n}} .
$$

On a ainsi défini une opération de l'ensemble $\{+,-\}^{*}$ des suites finies de signes sur l'ensemble des intervalles standard. 
Dans la suite, on note $\mathbb{L}_{0}^{+}$(resp. $\mathbb{L}_{0}^{-}$) l'intervalle standard $(0,1)$ (resp. l'intervalle standard $(1,0)$ ) où 1 est considéré comme un mot de Christoffel positif (resp. négatif) et on pose $\mathbb{L}_{0}=\mathbb{L}_{0}^{-} \cup \mathbb{L}_{0}^{+}$; c'est la liste ordonnée lexicographiquement de tous les mots de Christoffel primitifs.

Proposition 2. Les intervalles standard sont les intervalles de la forme $\mathbb{L}_{0}^{\omega}, \omega$ parcourant l'ensemble $\{+,-\}^{*}$ des suites finies de signes. Les mots de Christoffel primitifs distincts de 0 et 1 sont les mots médians des intervalles standard.

Preuve. Il suffit de prouver que les intervalles standard positifs sont les intervalles de la forme $\mathbb{L}_{0}^{+\omega}, \omega$ parcourant l'ensemble $\{+,-\}^{*}$ des suites finies de signes et que les mots de Christoffel primitifs positifs distincts de 0 et de 1 sont les mots médians des intervalles standard positifs. Tout d'abord, il est clair que les mots médians des intervalles standard de la forme $\mathbb{L}_{0}^{+\omega}, \omega$ parcourant $\{+,-\}^{*}$, sont des mots de Christoffel primitifs positifs. Montrons qu'il n'y en a pas d'autres (sauf 0 et 1 ) : soit $m$ un mot de Christoffel primitif positif distinct de 0 et 1 ; posons $f_{0}=0, g_{0}=1$; si $m \neq 01$ alors ou bien $m \in \mathbb{L}_{0}^{+-}$et on pose $f_{1}=f_{0}, g_{1}=f_{0} g_{0}$, ou bien $m \in \mathbb{L}_{0}^{++}$et on pose $f_{1}=f_{0} g_{0}, g_{1}=g_{0}$. On construit ainsi par récurrence deux suites $\left(f_{n}\right)$ et $\left(g_{n}\right)$ telles que pour tout $n \geq 1$, on ait $m \in\left(f_{n}, g_{n}\right)$ et $f_{n}=f_{n-1} g_{n-1}, g_{n}=g_{n-1}$ ou alors $f_{n}=f_{n-1}, g_{n}=f_{n-1} g_{n-1}$ pourvu que $m \neq f_{n-1} g_{n-1}$. Comme, d'après le corollaire 1 à la proposition 1 , la suite d'entiers $\left(\left|f_{n}\right|+\left|g_{n}\right|\right)$ est strictement croissante majorée par $|m|$, il existe un entier $N$ tel que $m=f_{N} g_{N}$. Maintenant, pour conclure, il suffit de noter que s'il y avait un intervalle standard positif distinct des $L^{+\omega}, \omega$ parcourant $\{+,-\}^{*}$, son mot médian admettrait deux factorisations standard distinctes, ce qui contredirait le théorème 1.

\section{Remarques.}

1. Quoique les intervalles ] $-\infty, n]$ et $[-n,+\infty[$, pour $n$ entier $\geq 1$, puissent être considérés comme des intervalles de Farey, les intervalles "lexicaux" correspondant $(1,011 \ldots 1)$ et $(1 \ldots 110,1)$ ne sont pas considérés comme standard.

2. Le procédé de construction des mots de Christoffel primitifs suggéré par la proposition 2 est analogue aux conditions de construction des mots sturmiens finis établies par Rauzy [14].

Corollaire 1. Deux intervalles standard qui ne sont pas contenus l'un dans l'autre ont au plus un mot en commum (qui est alors l'une de leurs extrémités). 
Corollaire 2. Un mot de Christoffel primitif $m$ qui appartient à l'intervalle standard $(f, g)$ s'écrit avec les lettres $f$ et $g$ (c'est-à-dire $\left.m \in\{f, g\}^{*}\right)$ et cette écriture est unique.

\section{Mots de Christoffel infinis}

\section{Mots de Christoffel infinis primitifs}

DÉFInitions. Étant donnés un mot infini $f$ et un entier $n \geq 1$, on note $\left.f\right|_{n}$ le mot fini constitué des $n$ premières lettres de $f$. On dit qu'un mot infini $f$ de 0 et de 1 est un mot de Christoffel primitif infini positif (resp. négatif) si pour tout $m \in \mathbb{N}$ il existe $n \geq m$ tel que $\left.f\right|_{n}$ soit un mot de Christoffel primitif positif (resp. négatif); leur ensemble est noté $\mathbb{L}^{+}$(resp. $\mathbb{L}^{-}$) et on pose $\mathbb{L}=\mathbb{L}^{-} \cup \mathbb{L}^{+}$.

Conventions. Désormais $\mathbb{L}^{+}$et $\mathbb{L}_{0}^{+}$(resp. $\mathbb{L}^{-}$et $\mathbb{L}_{0}^{-}$) sont munis de l'ordre lexicographique induit par $0<1$ (resp. $1<0$ ) ; les ensembles $\mathbb{L}_{0} \backslash\{0,1\}$ et $\mathbb{L}$ sont ainsi partiellement ordonnés, deux mots étant comparables s'ils sont tous deux positifs ou tous deux négatifs.

On rappelle enfin que pour tout $u \in X^{*}, u^{\infty}$ désigne le mot infini périodique de période $u$.

Identifications. Étant donnés un mot de Christoffel primitif fini $f$ et un entier positif $n$, le chemin associé au mot $f_{\gamma} f^{n-1} f_{\delta}$ est celui qui approche au plus près sans l'atteindre le segment joignant les extrémités du chemin de $f^{n}$; de même, à la limite, le chemin associé au mot $f_{\gamma} f^{\infty}$ est celui qui approche au plus près sans l'atteindre la demi-droite de pente $\rho(f)$. L'application

$$
\begin{gathered}
\mathbb{L}_{0} \backslash\{0,1\} \rightarrow \mathbb{L} \\
f \mapsto f_{\gamma} f^{\infty}
\end{gathered}
$$

est un plongement d'ensembles ordonnés qui permet d'identifier les mots de Christoffel primitifs finis distincts de 0 et 1 à des éléments de $\mathbb{L}$; il est également commode et cohérent d'identifier $0 \in \mathbb{L}_{0}$ avec $10^{\infty} \in \mathbb{L}^{-}$et $1 \in \mathbb{L}_{0}$ avec $01^{\infty} \in \mathbb{L}^{+}$, étant bien entendu que 0 et 1 restent les deux seuls mots de Christoffel pouvant être considérés comme négatifs ou positifs. Dorénavant les éléments de $\mathbb{L}$ appartenant à l'image de $\mathbb{L}_{0}$ seront représentés par leur écriture de mot de Christoffel primitif fini. Même avec cette nouvelle convention d'écriture, l'ordre de $\mathbb{L}^{+}$(resp. $\mathbb{L}^{-}$) reste l'ordre lexicographique induit par $0<1$ (resp. $1<0$ ), comme le prouve la : 
Proposition 3. Soit $f \in \mathbb{L}_{0}$; si $f=0$ on pose $f_{\gamma}=1$ et si $f=1$, $f_{\gamma}=0$. L'ensemble des mots de Christoffel primitifs finis ou infinis qui sont lexicographiquement plus grands que $f_{\gamma} f^{\infty}$ admet un plus petit élément qui est $f$.

Preuve. Il est clair que $f_{\gamma} f^{\infty}<f$. Soit $m$ un mot de Christoffel primitif fini ou infini tel que $f_{\gamma} f^{\infty}<m \leq f$. Si $m \in \mathbb{L}_{0}$, c'est un mot des intervalles standard $\left(f_{\gamma} f^{n}, f\right)$ pour tout $n \in \mathbb{N}$; en particulier si $n>|m|$, c'est l'unique mot de l'intervalle standard $\left(f_{\gamma} f^{n}, f\right)$ ayant moins de $n$ lettres, c'est-à-dire $f$. Si au contraire $m$ était un mot de Christoffel infini, alors il admettrait un préfixe fini plus grand que tous les $f_{\gamma} f^{n}, n \in \mathbb{N}$, et donc un préfixe de Christoffel fini appartenant à tous les intervalles standard $\left(f_{\gamma} f^{n}, f\right)$; ce préfixe ne pourrait donc être que $f$, ce qui est impossible.

Corollaire. Soient $u$ et $v \in \mathbb{L}$ tels que $u<v$; il existe alors $f \in \mathbb{L}_{0}$ tel que $u<f<v$.

Preuve. Il suffit de prendre pour $f$ un préfixe fini de $v$ qui est un mot de Christoffel plus grand que $u$.

\section{Suites de signes}

On considère désormais que les intervalles standard $(f, g)$ sont complétés par les mots de Christoffel primitifs infinis $m$ tels que $f<m<g$.

Soit $f$ un mot de Christoffel primitif fini. Si $f=1$, alors les intervalles standard contenant $f$ sont les intervalles de la forme $\mathbb{L}^{++\cdots+}$ ou $\mathbb{L}^{-\cdots \cdots-}$. Si $f=0$, les intervalles standard qui le contiennent sont les intervalles de la forme $\mathbb{L}^{+-\cdots-}$ et $\mathbb{L}^{-++\cdots+}$. Si $f \neq 0,1$, alors, d'après la proposition 2, $f$ est le mot médian d'un intervalle standard unique $I=\mathbb{L}^{\omega_{1} \omega_{2} \cdots \omega_{n}}$ et les intervalles standard qui contiennent $f$ sont les intervalles de la forme $I^{+--\cdots-}=\left(f, f^{n} f_{\delta}\right)$ et $I^{-++\cdots+}=\left(f_{\gamma} f^{n}, f\right)$ pour $n$ parcourant $\mathbb{N}$.

Soit maintenant $f \in \mathbb{L} \backslash \mathbb{L}_{0}$. Pour tout intervalle standard $I$ contenant $f$, on a $f \in I^{+} \cap I^{-}$si bien qu'il existe une unique suite infinie $\omega=$ $\omega_{1} \omega_{2} \cdots \omega_{n} \cdots$ de signes $\omega_{i}= \pm$ telle que, pour tout $n \in \mathbb{N}, f \in \mathbb{L}^{\left.\omega\right|_{n}}=$ $\mathbb{L}^{\omega_{1} \omega_{2} \cdots \omega_{n}}$; la suite $\omega$ ne peut être ultimement constante sous peine que $f \in \mathbb{L}_{0}$. Réciproquement, étant donnée une suite infinie de signes $\omega=$ $\omega_{1} \omega_{2} \cdots \omega_{n} \cdots$ non ultimement constante, l'intervalle standard $\mathbb{L}^{\left.\omega\right|_{n}}$ ne peut contenir de mot de Christoffel primitif fini $g$ de longueur $|g|<n$, d'après le corollaire 1 à la proposition 1 ; donc l'intersection des $\mathbb{L}^{\left.\omega\right|_{n}}$ ne contient pas de mot de $\mathbb{L}_{0}$ et, d'après le corollaire à la proposition 3 , elle contient un unique mot de $\mathbb{L}$ qui est d'ailleurs facile à construire. Posons, pour tout 
$n \geq 1, \mathbb{L}^{\left.\omega\right|_{n}}=\left(a_{n}, b_{n}\right)$ avec $a_{n}, b_{n} \in \mathbb{L}_{0} ;$ si $\omega_{n+1}=-$, alors $a_{n+1}=a_{n}$ et $b_{n+1}=a_{n} b_{n}$, si $\omega_{n+1}=+$ alors $a_{n+1}=a_{n} b_{n}$ et $b_{n+1}=b_{n}$; pour tout $m \leq n, a_{n} \in \mathbb{L}^{\left.\omega\right|_{m}}$ et la suite $\left(a_{n}\right)$ détermine un mot de $\mathbb{L}$ qui est dans l'intersection des $\mathbb{L}^{\left.\omega\right|_{n}}$. Résumons ce qu'on vient d'établir :

ThÉORÈme 2. Pour tout $f \in \mathbb{L} \backslash \mathbb{L}_{0}$ il existe une unique suite infinie $\omega(f)$ de signes + et - non ultimement constante telle que les intervalles standard qui contiennent $f$ soient les $\mathbb{L}^{\left.\omega(f)\right|_{n}}, n$ parcourant $\mathbb{N}^{*}$. Réciproquement si $\omega$ est une suite infinie non ultimement constante de + et de - l'intersection des intervalles standard $\mathbb{L}^{\left.\omega\right|_{n}}$ est réduite à un élément de $\mathbb{L} \backslash \mathbb{L}_{0}$.

DÉfinition. Avec les notations du théorème on dit que $\omega(f)$ est la suite de signes de $f$.

\section{Remarque.}

La construction de $f \in \mathbb{L} \backslash \mathbb{L}_{0}$ à partir de $\omega(f)$ est équivalente aux conditions de construction des mots sturmiens établies par Rauzy [14].

Corollaire 1. Soit $h$ un mot de Christoffel primitif fini ou infini appartenant à l'intervalle standard $(f, g)$. Tout préfixe assez long de $h$ qui est un mot de Christoffel appartient au monoïde libre $\{f, g\}^{*}$ et sa factorisation en $f$ et $g$ est unique.

Preuve. Compte tenu du corollaire 2 à la proposition 2 , c'est une conséquence immédiate du théorème.

Corollaire 2 ét Définition. Soit $f \in \mathbb{L} \backslash \mathbb{L}_{0}$ et soit $-f$ le mot de Christoffel opposé c'est-à-dire celui dont la suite des signes se déduit de celle de $f$ en remplaçant + par - et inversement. Alors les facteurs finis de $-f$ sont les facteurs finis de $f$ lus à l'envers.

Preuve. Il suffit de constater que, si on note $\bar{F}=-$ et $\overline{=}=+$ on a l'équivalence $(f, g)=\mathbb{L}^{\omega_{1} \omega_{2} \cdots \omega_{n}} \Leftrightarrow(-g,-f)=\mathbb{L}^{\overline{\omega_{1}} \overline{\omega_{2}} \cdots \overline{\omega_{n}}}$ (pour $f$ et $g \in$ $\left.\mathbb{L}_{0}\right)$.

\section{Pentes des mots de Christoffel infinis}

Remarquons d'abord que pour tout $f \in \mathbb{L}_{0}$, la suite des

$$
r\left(f_{\gamma} f^{n}\right)=\frac{\left|f_{\gamma}\right|_{1}+n|f|_{1}}{\left|f_{\gamma}\right|_{0}+n|f|_{0}}
$$

converge en croissant vers $r(f)$. 
Proposition 4 et Définition. Soit $f$ un mot de Christoffel primitif infini. La suite $\left(\rho_{n}\right)=\left(\rho\left(\left.f\right|_{n}\right)\right)$ des pentes des préfixes de $f$ est une suite convergente de nombres rationnels. Sa limite $\rho(f)$ s'appelle la pente de $f$.

Preuve. Il suffit d'établir le résultat pour les mots de Christoffel positifs $f$. Parmi les préfixes $\left.f\right|_{n}$ de $f$, soient $\left.f\right|_{n_{k}}$ ceux qui sont des mots de Christoffel primitifs ; la sous-suite $\left(\rho_{n_{k}}\right)$ de $\left(\rho_{n}\right)$ est croissante majorée par $\rho(g)$ pour tout $g \in \mathbb{L}_{0}$ tel que $f<g$. Soient enfin $m \in \mathbb{N}$ et $n_{k} \geq m$; on a, par définition des mots de Christoffel primitifs :

$$
\rho_{n_{k}}>\rho_{m}>\rho_{n_{k}}-\frac{1}{\left|f_{\left.\right|_{n_{k}}}\right|_{0}}=\rho_{n_{k}}-\frac{1+\rho_{n_{k}}}{n_{k}}
$$

ce qui achève la preuve.

Intervalles de Farey. On rappelle qu'un intervalle de Farey est un intervalle de la forme $\left[\frac{p}{q}, \frac{r}{s}\right]$ où les nombres rationnels $\frac{p}{q}$ et $\frac{r}{s}$, écrits sous forme canonique, sont tels que $q r-p s=+1$. Si $I=\left[\frac{p}{q}, \frac{r}{s}\right]$ est un intervalle de Farey alors $I^{-}=\left[\frac{p}{q}, \frac{p+r}{q+s}\right]$ et $I^{+}=\left[\frac{p+r}{q+s}, \frac{r}{s}\right]$ sont aussi des intervalles de Farey ; pour plus de détails sur les intervalles de Farey, voir par exemple [8], ch. III. Ajoutons que $[0, \infty]=\mathbb{R}^{+} \cup\{\infty\}$ et $[\infty, 0]=\mathbb{R}^{-} \cup\{\infty\}$ sont considérés comme des intervalles de Farey de la droite projective réelle $\mathbb{P}^{1}$. En s'inspirant de la théorie précédente et en notant $[0, \infty]=\left(\mathbb{P}^{1}\right)^{+}$, $[\infty, 0]=\left(\mathbb{P}^{1}\right)^{-}$, on montre que les intervalles de Farey s'écrivent $\left(\mathbb{P}^{1}\right)^{\omega}$ où $\omega$ est une suite finie de + et de - et donc que tout nombre réel irrationnel possède "un développement de Farey" qui est l'analogue des suites de signes des mots de Christoffel primitifs infinis et qui se déduit très simplement de son développement en fraction continue. Ce "développement de Farey" est presque identique au développement en "suites L-R" de G. N. Raney [13], mais la présentation ci-dessus est due à J. Riss, [15].

Proposition 5. La fonction pente $\rho$ est une bijection croissante de $\mathbb{L}$ sur la droite projective réelle $\mathbb{P}^{1}$ dont la bijection réciproque est

$$
\rho^{-1}(\alpha)=\left\{\begin{array}{l}
\left(<n \frac{\alpha}{1+\alpha}>-<(n-1) \frac{\alpha}{1+\alpha}>\right) \text { si } \alpha>0, \\
\left(1-<n \frac{1}{1-\alpha}>+<(n-1) \frac{1}{1-\alpha}>\right) \text { si } \alpha<0,
\end{array}\right.
$$

où $\langle x\rangle$ désigne $[x]$ si $x \notin \mathbb{N}^{*}$ et $x-1$ si $x \in \mathbb{N}^{*}$.

Preuve. L'image par $\rho$ de l'intervalle standard $\mathbb{L}^{\omega}$ avec $\omega \in\{+,-\}^{*}$ est l'intervalle de Farey rationnel $\mathbb{P}^{1}(\mathbb{Q})^{\omega}$ parce que $\rho(0)=0, \rho(1)=\infty$ et 
que la pente du mot médian de l'intervalle standard $(f, g)$ est le terme médian de l'intervalle de Farey $[\rho(f), \rho(g)]$. Il en résulte que si $f$ est un mot de Christoffel primitif infini de suite de signes $\omega=\omega_{1} \omega_{2} \cdots \omega_{n} \cdots$, alors $\{\rho(f)\}=\bigcap_{n \geq 1}\left(\mathbb{P}^{1}\right)^{\omega_{1} \omega_{2} \cdots \omega_{n}}$, autrement dit $\omega$ est aussi le développement de Farey de $\rho(f)$. Par suite $\rho$ est une bijection de $\mathbb{L}$ sur $\mathbb{P}^{1}$ telle que pour tous mots de Christoffel primitifs $f$ et $g$ on ait : $f<g \Rightarrow \rho(f)<\rho(g)$.

Enfin, pour établir la formule de la bijection réciproque, supposons d'abord $\alpha>0$. Soit $f_{\alpha}=\rho^{-1}(\alpha)$ le mot de Christoffel primitif de pente $\alpha ; f_{\alpha}$ correspond au chemin situé strictement au-dessous de la demidroite $y=\alpha x(x>0)$, mais qui l'approche au plus près ; ainsi $f_{\alpha}=$ $\begin{array}{llllllllll}0 & 1^{m_{1}} & 0 & 1^{m_{2}} & 0 & \cdots & 0 & 1^{m_{k}} & 0 & \cdots\end{array}$ avec $m_{k}=\langle\alpha k\rangle-\langle\alpha(k-1)\rangle$, étant entendu que $1^{m_{k}}$ est le mot vide si $m_{k}=0$; écrivons $f_{\alpha}=a_{1} a_{2} \cdots a_{k} \cdots$ et considérons la substitution $s: s(0)=0, s(1)=01$; l'image par $s$ de tout préfixe $h$ de $f_{\alpha}$ a pour pente $\frac{h_{1}}{h_{0}+h_{1}}$ soit $\frac{\rho(h)}{1+\rho(h)}$. Il en résulte que

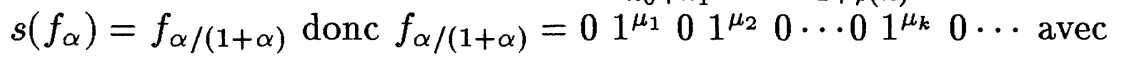

$$
\mu_{k}=<\frac{\alpha k}{\alpha+1}>-<\frac{\alpha(k-1)}{\alpha+1}>
$$

d'une part et

$$
f_{\alpha /(1+\alpha)}=s\left(a_{1}\right) s\left(a_{2}\right) \cdots s\left(a_{k}\right) \cdots
$$

d'autre part, d'où :

$$
s\left(a_{k}\right)=01^{\mu_{k}} \text { ou encore } a_{k}=\mu_{k} .
$$

Compte-tenu de la convention de lecture d'un chemin de pente négative qui consiste à renverser l'ordre, changer $\alpha$ en $-\alpha$ dans le raisonnement précédent revient à inverser les pentes tout en substituant 0 à 1 et 1 à 0 ; ce qui donne le mot de Christoffel $\rho^{-1}(\alpha)$ dans ce cas.

Exemple [2]. Le point fixe de la substitution $0 \rightarrow 01,1 \rightarrow 011$ est $f=$ $01011010110110101101011 \cdots$, sa suite de signes est $\omega(f)=++-+-+$ - $\cdots$ et pour tout $n \geq 1$, la $n$-ième lettre de $f$ est

$$
\left[n \frac{\sqrt{5}-1}{2}\right]-\left[(n-1) \frac{\sqrt{5}-1}{2}\right] \text {. }
$$

Règle de conversion. Soit $x$ un nombre réel positif irrationnel donné avec son développement en fraction continue, $x=\left[a_{0}, a_{1}, \cdots, a_{n}, \cdots\right],\left(a_{0}=\right.$ $[x])$, et soit $f$ le mot de Christoffel de pente $x$. La suite des signes de $f$ est

$$
\omega(f)=+{ }^{a_{0}+1}-{ }^{a_{1}}+{ }^{a_{2}} \cdots+{ }^{a_{2 k}}-{ }^{a_{2 k+1}} \cdots \text {. }
$$


Nous prouverons cette cette règle de conversion un peu plus loin.

\section{Substitutions de mots de Christoffel}

\section{Substitutions standard}

On rappelle (voir le corollaire 1 au théorème 2 ) que si $m$ est un mot de Christoffel primitif fini ou infini de l'intervalle standard $(f, g)$, alors tout préfixe assez long de $m$, qui est un mot de Christoffel, s'écrit de manière unique avec les lettres $f$ et $g$.

Définitions. Soit $(f, g)$ un intervalle standard et soient $f^{\prime}, g^{\prime} \in X^{*}$. En substituant à $f$ et $g$ respectivement $f^{\prime}$ et $g^{\prime}$, on définit une application $\sigma$ de $(f, g)$ dans $X^{*}$ : si h est un mot de Christoffel primitif de $(f, g)$, l'image de $h$ par $\sigma$ est l'unique mot admettant pour préfixes les $\sigma(m)$ où $m$ parcourt l'ensemble des préfixes de $h$ qui sont des mots de Christoffel mais qui ne sont pas préfixes de $f ; \sigma(h)$ est donc lui même un mot de Christoffel primitif dans le cas où $f^{\prime}$ et $g^{\prime}$ sont des mots de Christoffel finis tels que $\left(f^{\prime}, g^{\prime}\right)$ soit un intervalle standard; dans ces conditions, on dit que $\sigma$ est une substitution standard croissante.

Étant donnée une application $\varphi$ d'un ensemble de mots de Christoffel primitifs sur un autre, on note $-\varphi$ l'application qui au mot de Christoffel $m$ associe le mot de Christoffel opposé $-\varphi(m)$ du mot de Christoffel primitif $\varphi(m)$. Étant donné un intervalle standard $(f, g)$, on dit qu'une application $\sigma$ de $(f, g)$ dans $X^{*}$ est une substitution standard décroissante si $-\sigma$ est une substitution standard croissante.

On note souvent $f^{\sigma}$ l'image $d u$ mot de Christoffel $f$ par la substitution standard $\sigma$.

Les substitutions standard jouissent des propriétés évidentes suivantes :

Proposition 6. Soient $J=(f, g)$ et $J^{\prime}=\left(f^{\prime}, g^{\prime}\right)$ deux intervalles standard. Il existe une unique bijection croissante (resp. décroissante) $\sigma$ de $J$ sur $J^{\prime}$ vérifiant $(u v)^{\sigma}=u^{\sigma} v^{\sigma}$ (resp. $(u v)^{\sigma}=v^{\sigma} u^{\sigma}$ ) pour tout intervalle standard $(u, v) \subset J$; c'est la substitution standard croissante (resp. décroissante) $\sigma$ telle que $f^{\sigma}=f^{\prime}$ et $g^{\sigma}=g^{\prime}$ (resp. $f^{\sigma}=g^{\prime}$ et $\left.g^{\sigma}=f^{\prime}\right)$. De plus, on a $\sigma\left(J^{+}\right)=J^{\prime+}$ et $\sigma\left(J^{-}\right)=J^{\prime-}$ si $\sigma$ est croissante, $\sigma\left(J^{+}\right)=J^{\prime-}$ et $\sigma\left(J^{-}\right)=J^{\prime+}$ si $\sigma$ est décroissante.

\section{Remarque.}

C'est la proposition 2 de [10] corrigée d'une erreur de notation. 
Corollaire. Soient $\alpha$ et $\beta \in\{+,-\}^{*}$ et soit $\sigma$ une substitution standard de $\mathbb{L}^{\alpha}$ sur $\mathbb{L}^{\beta}$. Pour tout $f \in \mathbb{L}^{\alpha} \backslash \mathbb{L}_{0}$, la suite des signes de $f$ s'écrit $\omega(f)=$ $\alpha \omega$; appelons $\bar{\omega}$ la suites de signes obtenue à partir de $\omega$ en changeant + en - et - en + . On a alors $\omega\left(f^{\sigma}\right)=\beta \omega$ si $\sigma$ est croissante et $\omega\left(f^{\sigma}\right)=\beta \bar{\omega}$ si $\sigma$ est décroissante.

Exemple. Soient $S(0 \rightarrow 1,1 \rightarrow 0)$ et $T(0 \rightarrow 01,1 \rightarrow 1)$ deux substitutions standard croissantes définies sur $\mathbb{L}^{+}$; en notant $I$ l'application identité, les substitutions standard de $\mathbb{L}^{+}$sur $\mathbb{L}^{+}$sont $I$ et $-S$, de $\mathbb{L}^{+}$sur $\mathbb{L}^{-}:-I$ et $S$, de $\mathbb{L}^{+}$sur $\mathbb{L}^{++}: T$ et $T \circ(-S) \ldots$

\section{Matrice d'une substitution standard}

Théorème 3 et Définition. Soit $\sigma$ une substitution standard définie sur un intervalle standard $J$. La matrice

$$
\left(\begin{array}{cc}
v_{1}^{\sigma} u_{0}-u_{1}^{\sigma} v_{0} & u_{1}^{\sigma} v_{1}-v_{1}^{\sigma} u_{1} \\
v_{0}^{\sigma} u_{0}-u_{0}^{\sigma} v_{0} & u_{0}^{\sigma} v_{1}-v_{0}^{\sigma} u_{1}
\end{array}\right)
$$

ne dépend pas du choix de l'intervalle standard $(u, v) \subset J$. On l'appelle matrice de la substitution $\sigma$ et on la note $H(\sigma)$. De plus det $H(\sigma)=+1$ si $\sigma$ est croissante et det $H(\sigma)=-1$ si $\sigma$ est décroissante.

\section{Remarques et notations.}

1. Au cas où l'un des mots $u, v, u^{\sigma}$ ou $v^{\sigma}$ est 1 , l'indétermination sur le symbole $1_{1}$ est levée par le fait que si $\sigma$ est croissante les intervalles $(u, v)$ et $\left(u^{\sigma}, v^{\sigma}\right)$ sont standard et si $\sigma$ est décroissante les intervalles $(u, v)$ et $\left(v^{\sigma}, u^{\sigma}\right)$ sont standard.

2. Pour tout couple de mots de Christoffel primitifs finis $(u, v)$, posons $M(u, v)=\left(\begin{array}{ll}v_{1} & u_{1} \\ v_{0} & u_{0}\end{array}\right)$. Si $(u, v)$ est un intervalle standard on a

$$
\operatorname{det}(M(u, v))=+1
$$

et, avec les notations du théorème,

$$
H(\sigma)=M\left(u^{\sigma}, v^{\sigma}\right) M(u, v)^{-1} .
$$

3. Dans le cas où $\sigma$ est définie sur $\mathbb{L}^{+}$sa matrice

$$
H(\sigma)=\left(\begin{array}{ll}
1_{1}^{\sigma} & 0_{1}^{\sigma} \\
1_{0}^{\sigma} & 0_{0}^{\sigma}
\end{array}\right)
$$


n'est autre que l'image dans $G L\left(\mathbb{Z}^{2}\right)$ par le foncteur d'abélianisation de l'endomorphisme du groupe libre engendré par $\{1,0\}$, déterminé par $1 \rightarrow 1^{\sigma}$ et $0 \rightarrow 0^{\sigma}$. (Nous devons cette remarque à Jacques Peyrière).

Preuve du Théorème 3. Pour tout intervalle standard $(u, v)$ contenu dans $J=(f, g)$, posons

$$
H(\sigma, u, v)=M\left(u^{\sigma}, v^{\sigma}\right) M(u, v)^{-1} .
$$

On a

$$
H(\sigma, u, u v)=H(\sigma, u, v)
$$

parce que

$$
M(u, u v)=M(u, v)\left(\begin{array}{cc}
1 & 0 \\
1 & 1
\end{array}\right)
$$

et

$$
M\left(u^{\sigma},(u v)^{\sigma}\right)=M\left(u^{\sigma}, v^{\sigma}\right)\left(\begin{array}{ll}
1 & 0 \\
1 & 1
\end{array}\right) ;
$$

de même $H(\sigma, u v, v)=H(\sigma, u, v)$ parce que

$$
M(u v, v)=M(u, v)\left(\begin{array}{ll}
1 & 1 \\
0 & 1
\end{array}\right),
$$

et

$$
M\left((u v)^{\sigma}, v^{\sigma}\right)=M\left(u^{\sigma}, v^{\sigma}\right)\left(\begin{array}{ll}
1 & 1 \\
0 & 1
\end{array}\right) .
$$

En particulier on a $H(\sigma, f, f g)=H(\sigma, f g, g)=H(\sigma, f, g)$, ce qui amorce une récurrence évidente permettant de conclure que $H(\sigma, u, v)$ ne dépend pas de l'intervalle standard $(u, v)$ contenu dans $(f, g)$. On note donc désormais $H(\sigma, f, g)=H(\sigma)$.

Soit $\varphi$ la substitution standard définie sur $\mathbb{L}^{+}$par $0^{\varphi}=f^{\sigma}$ et $1^{\varphi}=g^{\sigma}$; la matrice $H(\varphi)=M\left(f^{\sigma}, g^{\sigma}\right)$ a pour déterminant +1 si $f^{\sigma}<g^{\sigma}$ et -1 sinon. De même soit $\psi$ la substitution standard définie sur $\mathbb{L}^{+}$par $0^{\psi}=f$ et $1^{\psi}=g$; sa matrice $H(\psi)=M(f, g)$ a pour déterminant +1 . I en résulte que, suivant que la substitution standard $\sigma$ est croissante ou décroissante, on a :

$$
\operatorname{det} H(\sigma)=\operatorname{det} H(\varphi) \operatorname{det} H(\psi)^{-1}=+1 \text { ou }-1 \text {. }
$$




\section{Mots comédians}

Définition. Étant donné un intervalle standard $(f, g)$ distinct de $\mathbb{L}^{+}$et de $\mathbb{L}^{-}$, on sait qu'il existe $h \in \mathbb{L}_{0}$ tel que $f=h g$ ou $g=f h$; on note $h=f \wedge g$; on note également $0 \wedge 1=10$ et $1 \wedge 0=01$. On dit que $f \wedge g$ est le mot comédian de l'intervalle standard $(f, g)$.

Interprétation géométrique. $\mathrm{Si}(f, g)$ est un intervalle standard distinct de $\mathbb{L}^{+}$et de $\mathbb{L}^{-}$, alors l'un des deux intervalles $(f \wedge g, f)$ ou $(g, f \wedge g)$ est standard. L'interpétation géométrique du mot comédian est donc claire sur la figure suivante.

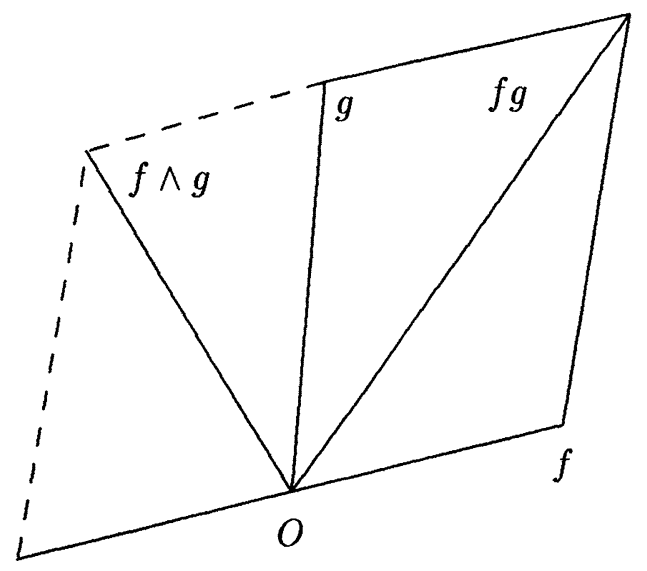

Figure 4

À la vue de l'interprétation géométrique, le résultat suivant est classique et fournit une définition équivalente du mot comédian : soit $(f, g)$ un intervalle standard, les nombres rationnels ou infinis $\rho(g), \rho(f), \rho(f g)$ et $\rho(f \wedge g)$ sont en division harmonique, autrement dit le birapport $(\rho(g), \rho(f), \rho(f g)$, $\rho(f \wedge g))$ vaut -1 .

Proposition 7. Soit $\left(\left(f_{n}, g_{n}\right)\right)$ une suite d'intervalles standard telle qu'on ait $f_{n+1}=f_{n}$ et $g_{n+1}=f_{n} \wedge g_{n}$ ou alors $f_{n+1}=f_{n} \wedge g_{n}$ et $g_{n+1}=g_{n}$. Il existe alors $n_{0} \in \mathbb{N}$ tel que $\left(f_{n_{0}}, g_{n_{0}}\right)=\mathbb{L}^{+}$ou $\mathbb{L}^{-}$.

Preuve. Tant que $\left(f_{n}, g_{n}\right)$ est différent de $\mathbb{L}^{+}$et de $\mathbb{L}^{-}$, la suite d'entiers positifs $\left(\left|f_{n}\right|+\left|g_{n}\right|\right)$ est strictement décroissante. 


\section{Homographies substitutives}

Définitions. Étant donnés $f$ et $g \in \mathbb{L}_{0}$ tels que $(f, g)$ ou $(g, f)$ soit un intervalle standard, on pose $f * g=f g$ si $(f, g)$ est standard et $f * g=g \wedge f$ si $(g, f)$ est standard ; on a aussi $0 * 1=01$ et $1 * 0=10$.

On dit qu'une bijection $\sigma$ de $\mathbb{L}$ sur $\mathbb{L}$ est une homographie substitutive croissante (resp. décroissante) si pour tout intervalle standard $(f, g)$ on a $\sigma(f g)=\sigma(f) * \sigma(g)($ resp. $\sigma(f g)=\sigma(g) * \sigma(f))$.

Exemple. On verra plus loin que la substitution $T$ appelée "translation +1 " définie sur l'intervalle standard $\mathbb{L}^{+}=(0,1)$ par $T(0)=01$ et $T(1)=1$ se prolonge de manière unique en une homographie substitutive par les substitutions standard suivantes : $T(1)=1$ et $T(10)=0$ sur $\mathbb{L}^{--}=$ $(1,10), T(10)=0$ et $T(0)=01$ sur $\mathbb{L}^{-+}=(10,0)$.

Proposition 8. Soient $\sigma$ une homographie substitutive et $J$ un intervalle standard. Alors au moins l'une des deux restrictions de $\sigma$ à $J^{+}$ou à $J^{-}$ est une substitution standard.

Preuve. Soit $I=(f, g)$. Adoptons la notation $\sigma(u)=u^{\sigma}$. Les mots de Christoffel $f^{\sigma}, g^{\sigma}$ et $(f g)^{\sigma}$ sont tous les trois positifs ou tous les trois négatifs. Supposons que $\sigma$ soit croissante (l'autre cas se traite de façon analogue). Si $(f g)^{\sigma}=f^{\sigma} g^{\sigma}$, c'est que $f^{\sigma}<(f g)^{\sigma}<g^{\sigma} ;$ si $(f g)^{\sigma}=g^{\sigma} \wedge f^{\sigma}$ alors ou bien $g^{\sigma}<f^{\sigma}<(f g)^{\sigma}$, ou bien $(f g)^{\sigma}<g^{\sigma}<f^{\sigma}$; autrement dit : ou bien la restriction de $\sigma$ à $J^{-}$est une substitution standard de $(f, f g)$ sur $\left(f^{\sigma},(f g)^{\sigma}\right)$ ou bien la restriction de $\sigma$ à $J^{+}$est une substitution standard de $(f g, g)$ sur $\left((f g)^{\sigma}, g^{\sigma}\right)$.

Proposition 9. Soit $\sigma$ une homographie substitutive. Il n'y a au plus qu'un nombre fini d'intervalles standard $J$ pour lesquels les restrictions de $\sigma$ à $J$ ne soient pas des substitutions standard. Ils sont totalement ordonnés par l'inclusion et le plus petit d'entre deux $I$ est tel que les extrémités de $I^{+}$ou de $I^{-}$soient $\sigma^{-1}(0)$ et $\sigma^{-1}(1)$.

Preuve. Tout d'abord si les restrictions de $\sigma$ à $\mathbb{L}^{+}$et à $\mathbb{L}^{-}$sont des substitutions standard, alors $\sigma^{-1}(0)=0$ ou 1 et $\sigma^{-1}(1)=0$ ou 1 ; d'où quatre éventualité : $\sigma=I$ (identité sur $\mathbb{L}), \sigma=-I, \sigma=S(0 \rightarrow 1,1 \rightarrow 0)$ et $\sigma=$ $-S$. Supposons maintenant que la restriction de $\sigma$ à $\mathbb{L}^{+}$ou à $\mathbb{L}^{-}$ne soit pas une substitution standard. Alors, d'après les propositions 7 et 8 , l'ensemble des intervalles standard $J$ pour lesquels les restrictions de $\sigma$ à $J$ ne soient pas des substitutions standard s'organise en une suite finie décroissante et si $I$ désigne le plus petit d'entre eux, alors nous sommes en présence de 
l'un des quatre cas suivants : $\sigma\left(I^{+}\right)=\mathbb{L}^{+}, \sigma\left(I^{+}\right)=\mathbb{L}^{-}, \sigma\left(I^{-}\right)=\mathbb{L}^{-}$ou $\sigma\left(I^{-}\right)=\mathbb{L}^{+}$.

Proposition 10 et Définition. Soit $\sigma$ une homographie substitutive et soient $J_{1}$ et $J_{2}$ deux intervalles standard. On suppose que les restrictions $\sigma_{1}$ de $\sigma$ à $J_{1}$ et $\sigma_{2}$ de $\sigma$ à $J_{2}$ sont des substitutions standard. Alors leurs matrices $H\left(\sigma_{1}\right)$ et $H\left(\sigma_{2}\right)$ ont même classe dans $P G L_{2}(\mathbb{Z})$; cette classe s'appelle par abus de langage la matrice de $\sigma$ et se note encore $H(\sigma)$.

Preuve. Dans le cas où $J_{1} \subset J_{2}$ ou $J_{2} \subset J_{1}$, ce résultat est déjà connu (théorème 3 ). Par récurrence, il suffit de prouver la proposition 10 dans les deux cas suivants :

a) $J_{1}=(u, u v), J_{2}=(v, u \wedge v)$ et les restrictions de $\sigma$ aux intervalles standard $(u, u \wedge v)$ et $(u v, v)$ ne sont pas des substitutions standard ;

b) $J_{1}=(u \wedge v, u), J_{2}=(u v, v)$ et les restrictions de $\sigma$ aux intervalles standard $(u \wedge v, v)$ et $(u, u v)$ ne sont pas des substitutions standard.

Supposons d'abord que $\sigma$ est croissante. On alors $(u v)^{\sigma}=v^{\sigma} \wedge u^{\sigma}$ et $(u \wedge v)^{\sigma}=v^{\sigma} u^{\sigma}$ et, dans le cas a $)$

$$
M(u, u v)=M(u, v)\left(\begin{array}{ll}
1 & 0 \\
1 & 1
\end{array}\right)
$$

et

$$
M\left(u^{\sigma}, v^{\sigma} \wedge u^{\sigma}\right)=M\left(u^{\sigma}, v^{\sigma}\right)\left(\begin{array}{cc}
-1 & 0 \\
1 & 1
\end{array}\right)
$$

parce que $u^{\sigma}=v^{\sigma}\left(v^{\sigma} \wedge u^{\sigma}\right)$. Il en résulte que

$$
H\left(\sigma_{1}\right)=M\left(u^{\sigma}, v^{\sigma}\right)\left(\begin{array}{cc}
-1 & 0 \\
0 & 1
\end{array}\right) M(u, v)^{-1} .
$$

De plus

$$
M(u, u \wedge v)=M(u, v)\left(\begin{array}{cc}
1 & 1 \\
-1 & 0
\end{array}\right)
$$

parce que $v=u(u \wedge v)$ et

$$
M\left(v^{\sigma}, v^{\sigma} u^{\sigma}\right)=M\left(u^{\sigma}, v^{\sigma}\right)\left(\begin{array}{ll}
1 & 1 \\
1 & 0
\end{array}\right) .
$$

Il en résulte que

$$
H\left(\sigma_{2}\right)=M\left(u^{\sigma}, v^{\sigma}\right)\left(\begin{array}{cc}
1 & 0 \\
0 & -1
\end{array}\right) M(u, v)^{-1},
$$


ce qui prouve la proposition dans ce cas.

Dans le cas b), on a

$$
M(u \wedge v, u)=M(u, v)\left(\begin{array}{cc}
0 & -1 \\
1 & 1
\end{array}\right)
$$

parce que $u=(u \wedge v) v$ et

$$
M\left(v^{\sigma} u^{\sigma}, u^{\sigma}\right)=M\left(u^{\sigma}, v^{\sigma}\right)\left(\begin{array}{ll}
0 & 1 \\
1 & 1
\end{array}\right)
$$

donc

$$
H\left(\sigma_{1}\right)=M\left(u^{\sigma}, v^{\sigma}\right)\left(\begin{array}{cc}
-1 & 0 \\
0 & 1
\end{array}\right) M(u, v)^{-1}
$$

De plus

$$
M(u v, v)=M(u, v)\left(\begin{array}{ll}
1 & 1 \\
0 & 1
\end{array}\right)
$$

et

$$
M\left(v^{\sigma} \wedge u^{\sigma}, v^{\sigma}\right)=M\left(u^{\sigma}, v^{\sigma}\right)\left(\begin{array}{cc}
1 & 1 \\
0 & -1
\end{array}\right)
$$

parce que $v^{\sigma}=\left(v^{\sigma} \wedge u^{\sigma}\right) u^{\sigma} ;$ donc

$$
H\left(\sigma_{2}\right)=M\left(u^{\sigma}, \dot{v}^{\sigma}\right)\left(\begin{array}{cc}
1 & 0 \\
0 & -1
\end{array}\right) M(u, v)^{-1}
$$

ce qui achève la preuve lorsque l'homographie substitutive $\sigma$ est croissante. Le cas où $\sigma$ est décroissante se ramène au précédent en remplaçant $\sigma$ par $-\sigma, H\left(\sigma_{1}\right)$ par

$$
\left(\begin{array}{cc}
-1 & 0 \\
0 & 1
\end{array}\right) H\left(\sigma_{1}\right)
$$

et $H\left(\sigma_{2}\right)$ par

$$
\left(\begin{array}{cc}
-1 & 0 \\
0 & 1
\end{array}\right) H\left(\sigma_{2}\right)
$$




\section{Homographies et substitutions}

THÉORÈme 4. Pour toute homographie substitutive $\sigma$ on a $\rho \circ \sigma=h_{\sigma} \circ \rho$ où $h_{\sigma}(x)=\frac{a x+b}{c x+d}$, la matrice $\left(\begin{array}{ll}a & b \\ c & d\end{array}\right)$ étant un représentant de $H(\sigma)$ dans $G L_{2}(\mathbb{Z})$.

Preuve. Interprétons, comme à l'accoutumée, la droite projective réelle comme l'ensemble des directions de droite dans $\mathbb{R}^{2}: \mathbb{P}^{1}=\left(\mathbb{R}^{2} \backslash\{(0,0)\}\right) / \mathbb{R}^{*}$ et la matrice $H=\left(\begin{array}{ll}a & b \\ c & d\end{array}\right)$ comme l'endomorphisme linéaire $\phi$ de $\mathbb{R}^{2}$ tel que $\phi(j)=a j+c i$ et $\phi(i)=b j+d i$ avec $j=(0,1)$ et $i=(1,0)$. Étant donnés deux vecteurs non nuls $V_{1}$ et $V_{2}$ de $\mathbb{R}^{2}$, si $H \in G L_{2}(\mathbb{Z})$, l'image dans $\mathbb{P}^{1}$ de $\phi\left(V_{1}+V_{2}\right)$ coïncide avec celle de $\phi\left(V_{1}\right)+\phi\left(V_{2}\right)$ ou bien avec celle de $\phi\left(V_{1}\right)-\phi\left(V_{2}\right)$ car det $H= \pm 1$; maintenant si $V_{1}=\left(x_{1}, y_{1}\right)$ et $V=\left(x_{2}, y_{2}\right)$ sont tels que $\rho\left(V_{1}\right)=\frac{y_{1}}{x_{1}}=\frac{p}{q}, \rho\left(V_{2}\right)=\frac{y_{2}}{x_{2}}=\frac{r}{s}$, nombres rationnels écrits sous forme canonique et vérifiant $q r-s p=1$, alors on a $\rho\left(V_{1}+V_{2}\right)=\frac{p+r}{q+s}$ et en posant $h_{\rho}\left(\frac{p}{q}\right)=\frac{P}{Q}, h_{\sigma}\left(\frac{r}{s}\right)=\frac{R}{S}$, on obtient

$$
h_{\sigma}\left(\frac{p+r}{q+s}\right)= \pm \frac{P+R}{Q+S} \quad \text { ou } \quad \pm \frac{P-R}{Q-S} .
$$

Autrement dit, pour tout intervalle standard $(f, g)$, on a, (en supposant pour fixer les idées que $\sigma$ est croissante), $h_{\sigma}(\rho(f g))=\rho\left(f^{\prime} g^{\prime}\right)$ ou $\rho\left(g^{\prime} \wedge f^{\prime}\right)$ où $f^{\prime}$ et $g^{\prime}$ sont tels que $\rho\left(f^{\prime}\right)=h_{\sigma}(\rho(f))$ et $\rho\left(g^{\prime}\right)=h_{\sigma}(\rho(g))$. D'autre part on a $\rho\left(0^{\sigma}\right)=\frac{b}{d}=h_{\sigma}(0)$ et $\rho\left(1^{\sigma}\right)=\frac{a}{c}=h_{\sigma}(\infty)$, la restriction de $\sigma$ à l'un ou l'autre des intervalles standard $\mathbb{L}^{+}$ou $\mathbb{L}^{-}$étant une substitution standard ; si cet intervalle-là contient $(f, g)$ et si $\rho\left(f^{\sigma}\right)=h_{\sigma}(\rho(f)), \rho\left(g^{\sigma}\right)=h_{\sigma}(\rho(g))$ avec $\rho(f)=\frac{p}{q}, \rho(g)=\frac{r}{s}$, alors on a

$$
\rho\left((f g)^{\sigma}\right)=\frac{a(p+r)+b(q+s)}{c(p+r)+d(q+s)}=h_{\sigma}(\rho(f g)),
$$

ce qui prouve par récurrence que $\rho \circ \sigma=h_{\sigma} \circ \rho \operatorname{sur}(f, g) \cap \mathbb{L}_{0}$ et même sur $\mathbb{L}_{0}^{+}$ou $\mathbb{L}_{0}^{-}$. Il en résulte que l'un des deux mots $(01)^{\sigma}$ et $(10)^{\sigma}$ et l'un d'eux seulement est le mot comédian de l'intervalle standard de bornes $0^{\sigma}$ et $1^{\sigma}$; la valeur correspondante $h_{\sigma}(\rho(01))$ ou $h_{\sigma}(\rho(10))$ vaut $\rho\left(\rho^{-1}\left(\frac{b}{d}\right) \wedge \rho^{-1}\left(\frac{a}{c}\right)\right)$ puisque la valeur $\rho\left(\rho^{-1}\left(\frac{b}{d}\right) \rho^{-1}\left(\frac{a}{c}\right)\right)$ est déjà prise et que $h_{\sigma} \circ \rho$ est une bijection de $\mathbb{L}_{0}$ sur $\mathbb{P}^{1}(\mathbb{Q})$. Si maintenant la restriction de $\sigma$ à l'intervalle standard $(f, f g)$ (ou bien $(f g, g)$, la preuve est la même) n'est pas une 
substitution standard et si $\rho\left(u^{\sigma}\right)=h_{\sigma}(\rho(u))$ pour $u=f, g$ et $f g$, on a alors :

$$
h_{\sigma}(\rho(f f g))=\rho\left((f g)^{\sigma} \wedge f^{\sigma}\right)=\rho\left((f f g)^{\sigma}\right)
$$

car la valeur : $\rho\left((f g)^{\sigma} f^{\sigma}\right)=\rho\left(\left(g^{\sigma} \wedge f^{\sigma}\right) f^{\sigma}\right)=\rho\left(g^{\sigma}\right)=h_{\sigma}(\rho(g))$ est déjà prise. Ceci achève de prouver par récurrence que $\rho \circ \sigma=h_{\sigma} \circ \rho$ sur $\mathbb{L}_{0}$. la preuve est tout à fait analogue lorsque $\sigma$ est décroissante. Enfin pour en déduire que $\rho \circ \sigma=h_{\sigma} \circ \rho$ sur $\mathbb{L}$ tout entier il suffit clairement d'utiliser d'une part le théorème des segments emboîtés et d'autre part la définition des mots de Christoffel primitifs infinis par leurs suites de signes.

Les corollaires qui suivent résultent immédiatement du théorème et éventuellement des propriétés les plus classiques du groupe modulaire $P S L_{2}(\mathbb{Z})$.

Corollaire 1 et DÉfinition. Pour toute substitution standard $\sigma$ il existe une unique homographie substitutive qui prolonge $\sigma$; on l'appelle le prolongement homographique de la substitution standard $\sigma$.

Corollaire 2. Les prolongements homographiques des substitutions standard forment, pour la composition des applications, un groupe isomorphe à $P G L_{2}(\mathbb{Z})$; les prolongements homographiques des substitutions standard croissantes en constituent un sous-groupe d'indice 2 isomorphe au groupe modulaire $P S L_{2}(\mathbb{Z})$.

Corollaire 3. $\grave{A}$ toute substitution standard croissante $\sigma$ on peut associer (de manière non unique) une suite finie d'entiers positifs $n_{1}, n_{2}$, $\ldots, n_{k}$ telle que $\sigma=T^{n_{1}} S T^{n_{2}} \ldots T^{n_{k-1}} S T^{n_{k}}$ où $S$ désigne la substitution $S(0)=1, S(1)=0$ et où $T$ désigne la substitution standard déjà définie par :

$$
\begin{aligned}
& T(0)=01 \text { et } T(1)=1 \text { sur l'intervalle standard }(0,1), \\
& T(1)=1 \text { et } T(10)=0 \text { sur l'intervalle standard }(1,10), \\
& T(10)=0 \text { et } T(0)=01 \text { sur l'intervalle standard }(10,0) .
\end{aligned}
$$

Corollaire 4. Les éléments d'ordre fini du groupe des prolongements homographiques des substitutions standard croissantes sont les conjugués de $S$ (c'est-à-dire les éléments de la forme $\sigma S \sigma^{-1}$ ) qui sont d'ordre 2 et les conjugués de $T S$ et de $T^{-1} S$ qui sont d'ordre 3. 
Corollaire 5. Soit $\sigma$ une substitution standard définie sur un intervalle standard $J \subset \mathbb{L}^{+}$(resp. $J \subset \mathbb{L}^{-}$) et soit $\left(\begin{array}{ll}a & b \\ c & d\end{array}\right)=H(\sigma)$. Pour que $\sigma$ se prolonge en une substitution standard sur $\mathbb{L}^{+}$(resp. sur $\left.\mathbb{L}^{-}\right)$il faut et il suffit que $a b \geq 0$ (resp. $a b \leq 0$ ).

\section{Remarque.}

Les prolongements homographiques des substitutions standard décroissantes sont de la forme $-\sigma=(-I) \circ \sigma$ où $\sigma$ est croissant ; il faut cependant prendre garde à ce que $(-I) \circ \sigma$ est en général différent de $\sigma \circ(-I)$ : $(-I) \circ S \circ(-I)=S$ mais $(-I) \circ T \circ(-I)=T^{-1}$.

Règle de conversion. Soit $a$ un nombre réel positif donné par son développement en fraction continue : $a=\left[a_{0}, a_{1}, a_{2}, \cdots, a_{n}, \cdots\right],\left(a_{0}=\right.$ $[a])$. La translation correspondant à la substitution standard croissante $T^{a_{0}}$ applique $\left[0, a_{1}, a_{2}, \cdots\right]$ sur $\left[a_{0}, a_{1}, a_{2}, \cdots\right]$ et $T^{a_{0}}$ applique $\mathbb{L}^{+}$sur $\mathbb{L}^{++^{a_{0}}}$; donc en notant $f_{n}$ le mot de Christoffel primitif de pente $\left[0, a_{n}, a_{n+1}, \cdots\right]$ et en notant $\omega\left(f_{n}\right)=+\omega_{n}$, on sait que $T^{a_{0}}$ transforme $+\omega_{1}$ en $++{ }^{a_{0}} \omega_{1}$. Pour tout $n \in \mathbb{N}^{*}$, l'homographie correspondant à la substitution standard décroissante $-S_{0} T^{a_{n}}$ applique $x$ sur $\frac{1}{a_{n}+x}$, donc $\left[0, a_{n+1}, a_{n+2}, \cdots\right]$ sur $\left[0, a_{n}, a_{n+1}, a_{n+2}, \cdots\right]$ et $-S_{0} T^{a_{n}}$ applique $\mathbb{L}^{+}$sur $\mathbb{L}^{+-^{a_{0}}}$, donc transforme $+\omega_{n+1}$ en $+-^{a_{n}} \frac{1}{\omega_{n+1}}$. Il en résulte que le mot de Christoffel primitif de pente $a$ a pour suite de signes $+{ }^{a_{0}+1}-{ }^{a_{1}}+{ }^{a_{2}} \cdots+{ }^{a_{2 k}}-{ }^{a_{2 k+1}} \cdots$.

\section{Applications arithmétiques}

Etant donné un mot de Christoffel primitif $f$, on rappelle que $\rho(f)$ désigne sa pente et $\omega(f)$ sa suite de signes ; de plus pour toute suite finie ou infinie $\omega$ de signes + et - on note $\bar{\omega}$ la suite obtenue en changeant + en - et vice-versa.

THÉORÈme 5. Pour tous $f, g \in \mathbb{L} \backslash \mathbb{L}_{0}$ les quatre assertions suivantes sont équivalentes :

(i) $g=f^{\sigma}$ où $\sigma$ est le prolongement homographique d'une substitution standard;

(ii) il existe $h \in \mathbb{L}^{+}$et deux substitutions standard $s$ et $t$ définies sur $\mathbb{L}^{+}$telles que $f=h^{s}$ et $g=h^{t}$

(iii) il existe $\alpha, \beta \in\{+,-\}^{*}$ et une suite infinie de signes $\omega$ telle que $\omega(f)=\alpha \omega$ et $\omega(g)=\beta \omega$ ou $\beta \bar{\omega}$; 
(iv) il existe $A, B, C, D \in \mathbb{Z}$ tels que $A D-B C= \pm 1$ et $A \rho(f) \rho(g)+$ $B \rho(f)+C \rho(g)+D=0$.

\section{Preuve.}

(i) $\Rightarrow$ (ii) : comme $f \notin \mathbb{L}_{0}$, il existe un intervalle standard $I$ contenant $f$ et sur lequel la restriction de $\sigma$ est une substitution standard. Soit $s$ la substitution standard croissante de $\mathbb{L}^{+}$sur $I$ et soit $h=f^{s^{-1}}$; alors $\sigma \circ s$ est une substitution standard définie sur $\mathbb{L}^{+}$telle que $g=h^{\sigma o s}$.

(ii) $\Rightarrow$ (iii) : soit $\eta$ la suite des signes de $h$. D'après le corollaire à la proposition 6 , il existe $\alpha, \beta \in\{+,-\}^{*}$ tels que la suite des signes de $h$ soit $\alpha \eta$ si $s$ est croissante ou $\alpha \bar{\eta}$ si $s$ est décroissante, et que la suite des signes de $h^{t}$ soit $\beta \eta$ si $t$ est croissante ou $\beta \bar{\eta}$ si $t$ est décroissante.

(iii) $\Rightarrow$ (iv) : quitte à remplacer $\alpha$ et $\beta$ par des préfixes plus longs, on peut supposer que $\omega=+\omega^{\prime}$; soient alors $s$ la substitution standard croissante de $\mathbb{L}^{+}$sur $\mathbb{L}^{\alpha}$ et $t$ la substitution standard de $\mathbb{L}^{+}$sur $\mathbb{L}^{\beta}$ croissante si $\omega(g)=\beta+\omega^{\prime}$ et décroissante si $\omega(g)=\beta-\bar{\omega}^{\prime}$. On a alors $f=u^{s}$ et $g=u^{t}$, où $u$ est le mot de Christoffel primitif tel que $\omega(u)=+\omega^{\prime}$. Soient maintenant $h_{s}$ et $h_{t}$ les homographies correspondant à $s$ et $t$ respectivement. Comme $g=f^{s^{-1} \text { ot }}$ on a $\rho(g)=h_{t} \circ h_{s}^{-1}(\rho(f))$ ce qui fournit une relation du type souhaité entre $\rho(f)$ et $\rho(g)$.

(iv) $\Rightarrow$ (i) : la relation $\rho(g)=\frac{-B \rho(f)-D}{A \rho(f)+C}$ avec $-B C+A D= \pm 1$ signifie, d'après le théorème 4 , que $g=f^{\sigma}$ où $\sigma$ est le prolongement homographique de la substitution standard de matrice $\left(\begin{array}{cc}-B & -D \\ A & C\end{array}\right)$.

Corollaire. Soit $f \in \mathbb{L} \backslash \mathbb{L}_{0}$. Les assertions suivantes sont équivalentes :

(i) $f=f^{\sigma}$ où $\sigma$ est le prolongement homographique d'une substitution standard non triviale;

(ii) il existe $h \in \mathbb{L}^{+}$et deux substitutions standard distinctes $s$ et $t$ définies sur $\mathbb{L}^{+}$telles que $f=h^{s}=h^{t}$;

(iii) $\omega(f)$ est une suite ultimement périodique ;

(iv) $\rho(f)$ est un nombre irrationnel quadratique.

Preuve. Dans le théorème 5 , le fait que $\sigma \neq i d$ dans (i) équivaut à $s \neq t$ dans (ii). Il suffit donc de montrer que l'assertion (iii) ci-dessus est équivalente à la suivante : il existe des mots finis $\alpha, \beta \in\{+,-\}^{*}$ et une suite infinie de signes $\omega$ tels que $\alpha \neq \beta$ et $\omega(f)=\alpha \omega=\beta \omega$ ou $\omega(f)=\alpha \omega=\beta \bar{\omega}$. 
Il est clair que, lorsque $\omega(f)$ est ultimement périodique, cette condition est réalisée. Réciproquement si $\alpha \omega=\beta \omega$ avec $\alpha \neq \beta$, alors $\alpha$ est un préfixe de $\beta$ ou le contraire; supposons par exemple que $\alpha=\beta \theta$ avec $\theta \in\{+,-\}^{*}$; alors $\omega=\theta \omega=\theta^{2} \omega=\cdots=\theta^{n} \omega=\cdots=\theta^{\infty}$. Enfin dans le cas où $\alpha \omega=\beta \bar{\omega}$, si $\alpha=\beta \theta$ avec $\theta \in\{+,-\}^{*}$ alors $\theta \omega=\bar{\omega}$ ou encore $\bar{\theta} \bar{\omega}=\omega$ donc $\omega=\bar{\theta} \theta \omega$ et comme précédemment $\omega=(\bar{\theta} \theta)^{\infty}$.

\section{Remarques.}

1. L'énoncé (ii) $\Leftrightarrow$ (iv) du corollaire est, au langage près, le théorème de S. Ito - S. Yasutomi ([9], Theorem 2.4) et de T. C. Brown [4].

2. Le fait que les nombres irrationnels quadratiques réels soient racines d'équations du type $A x^{2}+(B+C) x+D=0$ avec $A, B, C, D \in \mathbb{Z}$ tels que $A D-B C= \pm 1$ est bien connu ; mais, comme l'a remarqué J. Riss [15], il s'obtient élégamment grâce à la théorie précédente, de la façon suivante :

Soit $x$ une racine de l'équation

$$
a x^{2}+b x+c=0
$$

avec $a, b, c \in \mathbb{Z}$ tels que $b^{2}-4 a c$ soit positif et non carré parfait ; on note $y$ l'autre racine de cette équation. Soit $f$ (resp. $g$ ) le mot de Christoffel primitif de pente $x$ (resp. $y$ ). À tout intervalle standard $I$ contenant $f$ mais pas $g$, on associe la substitution standard croissante $\sigma_{I}$ de $\mathbb{L}^{+}$sur $I$; soit $\left(\begin{array}{ll}p & r \\ q & s\end{array}\right)$ sa matrice. Dans ces conditions la racine positive de l'équation :

$$
a(p X+r)^{2}+b(p X+r)(q X+s)+c(q X+s)^{2}=0
$$

n'est autre que la pente de $\sigma_{I}^{-1}(f)$ tandis que l'autre racine, obligatoirement négative, est la pente de $\sigma_{I}^{-1}(g)$. En écrivant l'équation $\left(\mathrm{E}_{2}\right)$ sous la forme :

$$
a_{I} X^{2}+b_{I} X+c_{I}=0
$$

avec $a_{I}, b_{I}, c_{I} \in \mathbb{Z}$, on calcule que $a_{I} c_{I}<0$ et que $b_{I}^{2}-4 a_{I} c_{I}=b^{2}-4 a c$. Comme il n'y a qu'un nombre fini de triplets d'entiers $\left(a_{I}, b_{I}, c_{I}\right)$ vérifiant ces conditions, il existe un intervalle standard $J \subset I$, sans bornes communes avec $I$, contenant $f$ mais pas $g$ et tel que l'équation $\left(\mathrm{E}_{3}\right)$ soit la même que :

$$
a_{J} X^{2}+b_{J} X+c_{J}=0
$$


On a donc $\sigma_{I}^{-1}(f)=\sigma_{J}^{-1}(f)$ ou encore $f=f^{\sigma}$ où $\sigma$ est le prolongement homographique de la substitution standard croissante qui applique $I$ sur $J$. En posant $I=(u, v)$ et $J=\left(u^{\sigma}, v^{\sigma}\right)$, la matrice de $\sigma$ est $\left(\begin{array}{ll}E & F \\ G & H\end{array}\right)$ avec :

$$
\begin{aligned}
& E=v_{1}^{\sigma} u_{0}-u_{1}^{\sigma} v_{0}, \\
& F=u_{1}^{\sigma} v_{1}-v_{1}^{\sigma} u_{1}, \\
& G=v_{0}^{\sigma} u_{0}-u_{0}^{\sigma} v_{0}, \\
& H=u_{0}^{\sigma} v_{1}-v_{0}^{\sigma} u_{1} .
\end{aligned}
$$

Comme $u<u^{\sigma}<v^{\sigma}<v$, on a

$$
E+H=v_{1}^{\sigma} u_{0}-v_{0}^{\sigma} u_{1}+u_{0}^{\sigma} v_{1}-u_{1}^{\sigma} v_{0} \geq 2,
$$

avec égalité si et seulement si :

$$
v_{1}^{\sigma} u_{0}-v_{0}^{\sigma} u_{1}=1 \text { et } u_{0}^{\sigma} v_{1}-u_{1}^{\sigma} v_{0}=1,
$$

c'est-à-dire si et seulement si les intervalles $\left(u, v^{\sigma}\right)$ et $\left(u^{\sigma}, v\right)$ sont standard, ce qui est impossible. Donc $E+H=\operatorname{Tr}(\sigma)>2$ : l'homographie associée à $\sigma$ est hyperbolique.

On a donc montré que l'équation

$$
a x^{2}+b x+c=0
$$

$\left(a, b, c\right.$ dans $\mathbb{Z}$, premiers entre eux dans leur ensemble, $b^{2}-4 a c>0, b^{2}-4 a c$ non carré parfait), peut se mettre sous la forme

$$
x=\frac{E x+F}{G x+H},
$$

$(E, F, G, H \in \mathbb{Z}, E H-F G=1, E+H>2)$.

Il existe alors un entier $\lambda$ tel que :

$$
G=\lambda a, H-E=\lambda b, F=-\lambda c,
$$

donc, en posant $\Delta=b^{2}-4 a c$, on obtient :

$$
\lambda^{2} \Delta=(H-E)^{2}+4 F G=(H+E)^{2}-4>0 .
$$

Autrement dit, pour tout entier $\Delta>0$ non carré parfait et congru à 0 ou 1 modulo 4, l'équation :

$$
X^{2}-\Delta Y^{2}=4
$$

possède une solution $(X, Y) \in \mathbb{Z}^{2}$ avec $X>2$. il en résulte que pour tout entier $D>0$ non carré parfait l'équation :

$$
X^{2}-D Y^{2}=1
$$

possède au moins une solution $(X, Y) \in \mathbb{Z}^{2}$ avec $X>1$. 


\section{BIBLIOGRAPHIE}

[1] J. BERNOULLI, Recueil pour les astronomes, Berlin, 1772.

[2] J. BERSTEL, Fibonacci words - a survey, in G. Rozenberg, A. Salomaa, ed., the Book of L, Springer Verlag (1986), 13-27.

[3] J.-P. BOREL, F. LAUBIE, Construction de mots de Christoffel, C. R. Acad. Sci. Paris, Série I 313 (1991), 483-485.

[4] T. C. BROWN, A characterisation of the quadratic irrationals, Canad. Math. Bull. 34 (1991), 36-41.

[5] T. C. BROWN, Descriptions of the characteristic sequence of an irrational, Canad. Math. Bull. 36 (1993), 15-21.

[6] D. CRISP, W. MORAN, A. POLLINGTON, P. SHIUE, Substitution invariant cutting sequences, Séminaire de Théorie des Nombres de Bordeaux, Série II, ce volume.

[7] E. B. CHRISTOFFEL, Observatio arithmetica, Annali di Matematica, Ser. 26 (1875), 145-152.

[8] G. H. HARDY, E. M. WRIGHT, An introduction to the theory of numbers, Oxford at the Clarendom Press, 4th ed., 1960.

[9] S. ITO, S. YASUTOMI, On continued fractions, substitutions and characteristic sequences $[n x+y]-[(n-1) x+y]$, Japan J. Math. 16 (1990), 287-306.

[10] F. LAUBIE, Prolongements homographiques des substitutions de mots de Christoffel, C. R. Acad. Sci. Paris 313 Série I (1991), 565-567.

[11] K. NISHIOKA, I. SHIOKAWA, J. TAMURA, Arithmetical properties of a certain power series, J. Number Theory 42 (1992), 61-87.

[12] D. PERRIN, Factorization of Free Monoids, in M. Lothaire, Combinatorics on words, Encyclopedia of Math. and its Applications 17, 63-99, Addison-Wesley, 1983.

[13] G. N. RANEY, On Continued Fractions and Finite Automata, Math. Ann. 206 (1973), 265-283.

[14] G. RAUZY, Mots infinis en arithmétique, in M. Nivat et D. Perrin ed., Automata on infinite words, Lecture Notes in Computer Science 192 (1985), 165-171.

[15] J. RISS, Communication personnelle, 1974.

[16] G. VIENNOT, Algèbres de Lie libres et Monoïdes libres, Lecture Notes in Math. 691 (1978).

J.-P. Borel, F. Laubie

Université de Limoges,

Faculté des Sciences, Département de Math.

123 avenue Albert Thomas

F-87060 Limoges Cedex 